\title{
Orientações sobre amamentação na atenção básica de saúde e associação com o aleitamento materno exclusivo
}

\author{
Guidance on breastfeeding in primary health care \\ and the association with exclusive breastfeeding
}

Jessica de Souza Alves ${ }^{1}$

Maria Inês Couto de Oliveira ${ }^{2}$

Rosane Valéria Viana Fonseca Rito ${ }^{3}$

${ }^{1}$ Programa de PósGraduação em Saúde

Coletiva, Instituto de Saúde Coletiva, Universidade Federal Fluminense (UFF) R. Marquês do Paraná 303/3o, Centro. 24030 210 Niterói RJ Brasil. jessicasalvess@hotmail.com ${ }^{2}$ Departamento de Epidemiologia e

Bioestatística. Instituto de Saúde Coletiva, UFF.

${ }^{3}$ Departamento de Nutrição e Dietética, Faculdade de Nutrição Emília de Jesus Ferreiro, UFF.

\begin{abstract}
This article aims to analyze the association between guidance on breastfeeding in primary health care and exclusive breastfeeding. It involved a cross-sectional study conducted in 2013 in the city of Rio de Janeiro, Brazil, by means of interviews with 429 mothers of infants below six months of age in primary care units. We estimated adjusted prevalence ratios by Poisson regression. In the final model, the variables associated with the outcome $(p \leq 0.05)$ were retained. The prevalence of exclusive breastfeeding was $50.1 \%$. In multivariate analysis, guidance on exclusive breastfeeding in primary care was directly associated with the outcome $(P R=1.32)$. Maternal income $<1$ minimum wage $(P R=0.77)$, previous breastfeeding experience for less than 6 months $(P R=0.73)$, not living with a partner $(P R=0.76)$, non-exclusive breastfeeding at discharge $(P R=0.78)$, alcohol consumption (OR $=0.57)$, guidance on pumping breast milk (PR $=0.53)$, pacifier use $(P R=0,74)$ and baby's age in months $(P R=0.78)$ were associated inversely with the outcome. Guidance on exclusive breastfeeding favored it, while inadequate guidance and practices were associated with a lower prevalence of the outcome.
\end{abstract}

Key words Breastfeeding guidance, Primary health care, Cross-sectional studies
Resumo $O$ objetivo deste artigo é analisar a associação entre o recebimento de orientaçães sobre amamentação na atenção básica à saúde e o aleitamento materno exclusivo. Estudo transversal conduzido em 2013, mediante entrevista a 429 mães de crianças menores de seis meses, em unidades básicas da cidade do Rio de Janeiro. Razões de prevalência ajustadas do desfecho foram obtidas por regressão de Poisson, sendo mantidas no modelo final as variáveis associadas ao desfecho $(p \leq 0,05)$. A prevalência de aleitamento materno exclusivo foi de 50,1\%. Na análise múltipla, a mãe receber orientação sobre aleitamento materno exclusivo na unidade básica se associou positivamente ao desfecho $(R P=1,32)$. Associaram-se negativamente ao desfecho: renda materna $<1$ salário mínimo $(R P=0,77)$, experiência prévia em amamentar por menos de 6 meses $(R P=0,73)$, não viver com companheiro $(R P=0,76)$, não amamentação exclusiva na alta hospitalar $(R P=0,78)$, consumo de bebida alcoólica $(R P=0,57)$, receber orientação sobre uso de bombas para ordenha das mamas ( $R P$ $=0,53)$, uso de chupeta $(R P=0,74)$ e idade crescente do bebê em meses $(R P=0,78)$. Ter recebido orientação sobre o aleitamento materno exclusivo contribuiu para o mesmo, enquanto orientações e práticas inadequadas se associaram a uma menor prevalência do desfecho.

Palavras-chave Aleitamento materno, Orientação, Atenção Primária à Saúde, Estudos transversais 


\section{Introdução}

Estudos evidenciam que o aleitamento materno confere inúmeros benefícios tanto para o bebê $\hat{e}^{1,2}$, como para a mãe $e^{3}$ A Organização Mundial da Saúde (OMS) recomenda o aleitamento materno exclusivo até os seis meses de vida, pois o leite materno reúne as características nutricionais ideais para a criança nesta fase da vida ${ }^{4}$.

No Brasil, a mediana de aleitamento materno exclusivo aumentou de 1,1 meses em 1996 para 1,4 meses em $2006^{5}$. Segundo pesquisa realizada nas capitais brasileiras e no Distrito Federal, constatou-se aumento de 1 mês na duração mediana do aleitamento materno exclusivo, que passou de 23,4 dias, em 1999, para 54,1 dias, em $2008^{6}$. Portanto, esta prática tem avançado, mas ainda está distante do preconizado ${ }^{4}$.

Os avanços na prática do aleitamento materno podem ser atribuídos às ações de promoção iniciadas no Brasil em 1981. Na década de 1990, estratégias de promoção do aleitamento materno nos serviços de saúde foram lançadas, como a Iniciativa Hospital Amigo da Criança pela OMS e UNICEF${ }^{8}$ e a Iniciativa Unidade Básica Amiga da Amamentação (IUBAAM), pela Secretaria de Saúde do Estado do Rio de Janeiro. Esta iniciativa preconiza "Dez Passos para o Sucesso da Amamentação", criados com base em revisão sistemática sobre ações desenvolvidas na atenção primária, com efetividade na extensão da duração do aleitamento materno exclusivo, como orientações prestadas às gestantes no pré-natal e às mães no acompanhamento do binômio mãefilho?.

Os índices e a duração do aleitamento materno exclusivo podem ser influenciados por intervenções de orientação e apoio ao aleitamento materno por profissionais de saúde ${ }^{10,11}$. Contudo, poucos estudos têm investigado as orientações prestadas às gestantes e mães na atenção básica, bem como sua associação com o aleitamento materno exclusivo.

O objetivo deste estudo foi analisar a associação entre o recebimento de orientações sobre amamentação em unidades básicas de saúde e o aleitamento materno exclusivo. Espera-se que as orientações prestadas às mães gerem um aumento na prevalência do desfecho estudado.

\section{Métodos}

Trata-se de estudo transversal conduzido em unidades básicas de saúde do Município do Rio de
Janeiro nos meses de novembro e dezembro de 2013, como parte da pesquisa "Avaliação dos fatores associados à doação de leite materno por usuárias de unidades básicas de saúde da cidade do Rio de Janeiro" ${ }^{\prime 2}$. A população do estudo original foi composta por mães de crianças menores de um ano acompanhadas pelas nove unidades básicas do município com "Posto de Recebimento de Leite Humano Ordenhado", que estimulam as nutrizes a doar o leite materno excedente e encaminham-no a Bancos de Leite Humano ${ }^{13}$.

$\mathrm{O}$ projeto de pesquisa foi desenhado para a investigação de vários desfechos relativos à amamentação e à doação de leite humano. O tamanho da amostra original foi definido com base em uma prevalência de $50 \%$ do desfecho, pois garante o maior tamanho de amostra possível para nível de erro e confiança controlados ${ }^{14}$. Considerando um alfa de $5 \%$ e um poder de $80 \%$, para detectar uma razão de chances de 1,5 , foi obtida uma amostra de 697 mães, a partir de uma demanda média mensal de 1321 bebês menores de um ano assistidos pelas nove unidades. A amostra de mães entrevistadas em cada unidade foi proporcional à média do número de crianças menores de um ano consultadas em cada unidade nos meses de setembro e outubro de 2013. Eram elegíveis mães com filho menor de um ano consultado pelo menos uma vez na unidade ${ }^{12}$.

No presente estudo foi investigada a associação entre as orientações recebidas sobre amamentação e o aleitamento materno exclusivo. Para tal, foram selecionadas apenas as mães de bebês menores de seis meses, em número de 429 . Com base em uma variável de exposição principal, o recebimento de orientações sobre as vantagens do aleitamento materno exclusivo até os seis meses de vida, o poder do estudo foi calculado em $69 \%$.

Os instrumentos foram questionários estruturados, previamente testados em estudo piloto realizado em duas unidades primárias da cidade do Rio de Janeiro, nos meses de setembro e outubro de 2013. Foram aplicados por seis entrevistadoras, enfermeiras ou nutricionistas, previamente capacitadas em treinamento com carga horária de 20 horas, e no estudo piloto. Sob supervisão, as entrevistadoras coletaram dados em todos os turnos de atendimento da unidade de saúde até que o tamanho amostral fosse atingido. O período de coleta de dados em cada unidade variou de 25 dias a um mês e meio.

As entrevistas foram conduzidas mediante assinatura de termo de consentimento livre e esclarecido, as mães sendo informadas sobre a 
não obrigatoriedade da participação no estudo, sendo garantido o sigilo das informações. O estudo foi submetido ao comitê de ética e pesquisa da Secretaria Municipal de Saúde do Rio de Janeiro e aprovado segundo o parecer no $228^{a}$, de 18/08/2013.

A alimentação do bebê foi verificada por meio de questões fechadas sobre o consumo nas últimas 24 horas (current status) de leite materno, água, chás, outros líquidos, outros tipos de leite e outros alimentos. Foi possível, dessa forma, tipificar se a criança recebeu ou não leite materno de forma exclusiva nas 24 horas que antecederam a pesquisa, segundo as definições da OMS ${ }^{4}$.

Foi construído um modelo teórico hierarquizado ${ }^{15}$ para a prática de aleitamento materno exclusivo, com base em revisão sistemática que identificou fatores que influenciam esta prática no cenário nacional ${ }^{16}$. No nível distal, foram contempladas características maternas e domiciliares; no nível intermediário, características da assistência pré-natal, ao parto, do bebê ao nascer e da assistência no hospital; e no nível proximal, as características de estilo de vida materno, as orientações em aleitamento materno recebidas na unidade básica de saúde e as características do bebê (Figura 1).
As variáveis de exposição principal foram as orientações em aleitamento materno. As orientações avaliadas tiveram como base procedimentos preconizados pela IUBAAM ${ }^{9}$ : orientar gestantes e mães sobre as vantagens do aleitamento materno exclusivo até os seis meses (relativa ao Passo $3)$; mostrar às gestantes e mães como amamentar e como manter a lactação, mesmo se vierem a ser separadas de seus filhos (Passo 6); encorajar a amamentação sob livre demanda (Passo 8); orientar gestantes e mães sobre os riscos do uso de mamadeiras (relativa ao Passo 9); implementar grupos de apoio à amamentação acessíveis a gestantes e mães (relativa ao Passo 10).

O banco de dados com as informações, obtidas por meio das entrevistas com as mães, foi elaborado por meio do programa Epi-Info 2000 e a análise dos dados foi conduzida pelo programa SPSS17.

Como a prevalência do desfecho foi elevada, optou-se por analisar os dados por modelo de regressão de Poisson com variância robusta ${ }^{17}$, sendo obtidas as razões de prevalência (RP) brutas e seus respectivos intervalos de confiança de $95 \%$. As variáveis de exposição que obtiveram p-valor menor ou igual a 0,20 foram selecionadas para análise múltipla. $\mathrm{O}$ modelo final foi composto

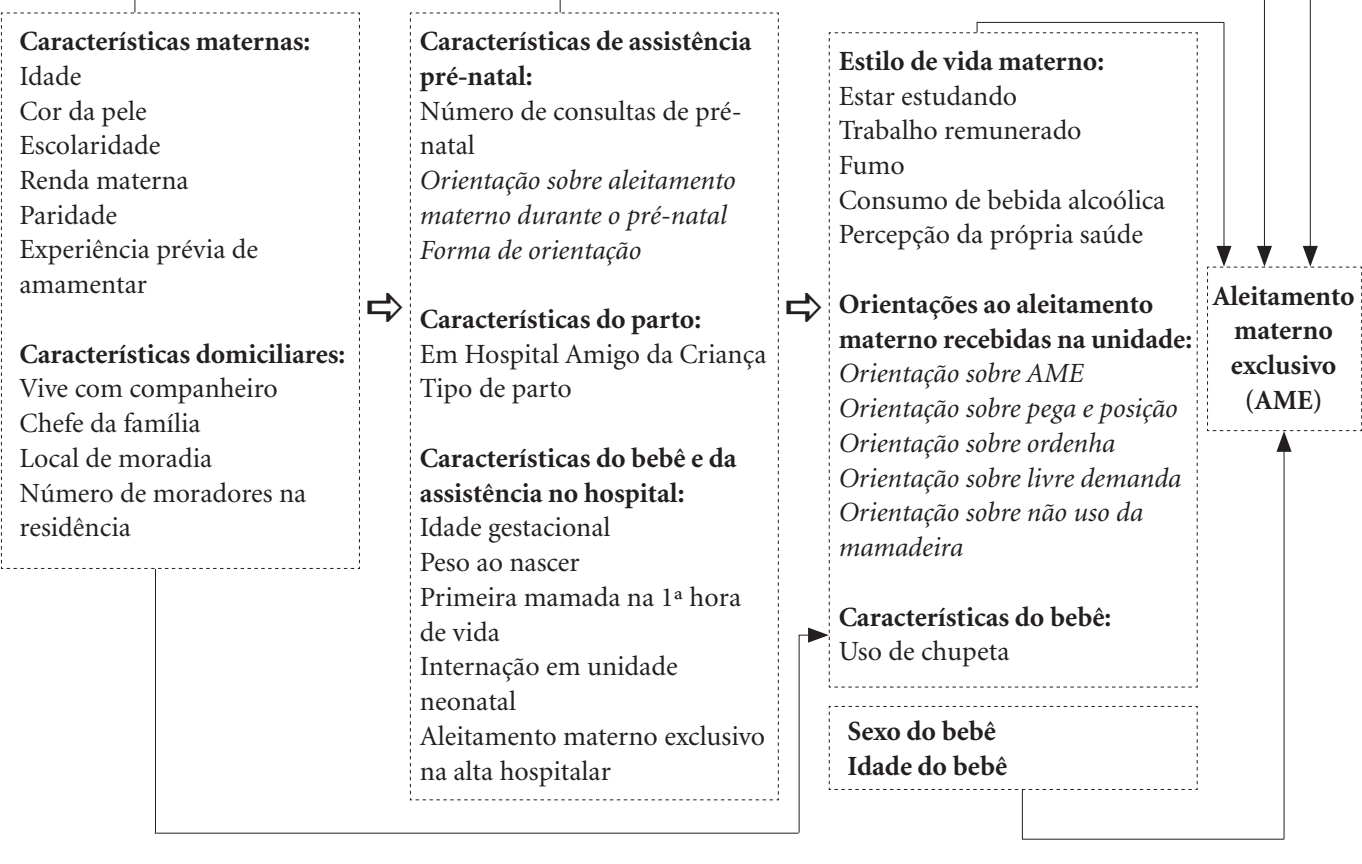

Figura 1. Modelo teórico hierarquizado da prática de aleitamento materno exclusivo entre mães usuárias de unidades básicas de saúde no município do Rio de Janeiro, 2013. 
pelas variáveis de exposição que se mostraram associadas ao desfecho com nível de significância observado menor ou igual a $5 \%(\mathrm{p} \leq 0,05)$.

\section{Resultados}

Das 697 mães de crianças menores de um ano amostradas, quatro se recusaram a ser entrevistadas e foram repostas por mães subsequentes, e duas foram duplamente entrevistadas, sendo a segunda entrevista descartada. Restaram 695 mães para análise, das quais 266 foram excluídas por terem filhos com seis meses ou mais. Para fins do presente estudo, foram analisadas 429 mães. A prevalência de aleitamento materno exclusivo entre as crianças menores de seis meses foi de $50,1 \%$.

Quanto às características maternas, 22,8\% das mães eram adolescentes, mais da metade tinha escolaridade inferior ao ensino médio completo, $28,0 \%$ recebiam menos de um salário mínimo, $38,2 \%$ não possuíam renda própria, e $13,5 \%$ das mães amamentaram o último filho por menos de seis meses. Quanto às características domiciliares, $21,2 \%$ das mães não viviam com o companheiro e $62,2 \%$ residiam em comunidade de baixa renda. Uma menor prevalência de aleitamento materno exclusivo $(\mathrm{p} \leq 0,20)$ foi observada entre mães adolescentes, não brancas, com escolaridade inferior ao ensino médio, com renda inferior a um salário mínimo, entre primíparas e entre multíparas que amamentaram o último filho por menos de seis meses, que não viviam com o companheiro e cujo chefe da família não era o companheiro (Tabela 1 ).

Em relação à assistência pré-natal, $10,7 \%$ foram acompanhadas por menos de seis consultas. Mais de três quartos das mulheres foi orientada sobre aleitamento materno, tendo $59,0 \%$ recebido orientações na consulta, $39,2 \%$ em grupos educativos e 4,4\% durante visita domiciliar. Quanto às características do parto, pouco mais de um quarto das mães tiveram seus filhos em Hospitais Amigos da Criança e 42,4\% tiveram parto cesáreo. Em relação às características do bebê e de sua assistência hospitalar, 6,1\% nasceram com baixo peso, $17,2 \%$ ficaram internados em unidade neonatal e $21,7 \%$ não tiveram alta hospitalar em aleitamento materno exclusivo. Uma menor prevalência de aleitamento materno exclusivo foi observada entre mães acompanhadas por menos de seis consultas pré-natais, com parto em hospitais não credenciados na Iniciativa Hospital Amigo da Criança e que não amamentavam exclusivamente na alta hospitalar (Tabela 2).

Quanto às varáveis proximais, $40,3 \%$ das mães exerciam trabalho remunerado, $12,4 \% \mathrm{fu}$ mavam, $11,7 \%$ consumiam bebida alcoólica e $15,4 \%$ tinham auto percepção desfavorável da saúde. A grande maioria das mães $(82,9 \%)$ foi orientada na unidade básica sobre a importância do aleitamento materno exclusivo por 6 meses, mais da metade $(63,4 \%)$ foi orientada sobre como colocar o bebê no peito para mamar, $56,4 \%$ sobre ordenha manual das mamas, $76,5 \%$ sobre livre demanda, e $76,9 \%$ quanto ao não uso de mamadeira. Mais da metade dos bebês $(55,1 \%)$ usava chupeta, e $41,0 \%$ estavam no segundo bimestre de vida. Uma menor prevalência de aleitamento materno exclusivo foi observada entre as mães que fumavam, que consumiam bebida alcoólica, que percebiam a própria saúde como ruim ou regular, que foram orientadas sobre ordenha das mamas com uso de bomba, cujos bebês usavam chupeta e não estavam no primeiro bimestre de vida. Uma maior prevalência de aleitamento materno exclusivo foi observada entre as mães que receberam orientações sobre a importância da amamentação exclusiva por 6 meses, sobre pega e posição do bebê no peito, sobre livre demanda e sobre o não uso de mamadeira (Tabela 3).

$\mathrm{Na}$ análise múltipla, ter recebido orientação sobre a importância da amamentação exclusiva por 6 meses associou-se positivamente ao aleitamento materno exclusivo. Já a renda materna inferior a um salário mínimo, a experiência prévia de amamentar o último filho por menos de seis meses, não viver com companheiro, não ter tido alta hospitalar em aleitamento materno exclusivo, consumo de bebida alcoólica, ter sido orientada sobre ordenha das mamas com uso de bomba, uso de chupeta pelo bebê e sua idade crescente em meses foram fatores associados negativamente ao aleitamento materno exclusivo ( $\mathrm{p}$ $\leq 0,05)$ (Tabela 4).

\section{Discussão}

A metade das crianças no primeiro semestre de vida, assistidas por unidades básicas que dispunham de Posto de Recebimento de Leite Humano Ordenhado, na cidade do Rio de Janeiro, estava em aleitamento materno exclusivo. Esta proporção foi superior à encontrada pela Pesquisa Nacional de Demografia e Saúde no Brasil, em $2006(38,6 \%)^{5}$, e pela Pesquisa de Prevalência de Aleitamento Materno nas Capitais Brasileiras e Distrito Federal 
Tabela 1. Prevalência e razão de prevalência bruta de aleitamento materno exclusivo em menores de 6 meses segundo características maternas e domiciliares. Município do Rio de Janeiro, 2013.

\begin{tabular}{|c|c|c|c|c|}
\hline Características & $\mathbf{N}$ & $\%$ & $\operatorname{AME}(\%)$ & $\mathrm{RPb}$ AME \\
\hline \multicolumn{5}{|l|}{ Idade da mãe } \\
\hline 13-19 anos & 98 & 22,8 & 39,8 & $0,748^{\star}$ \\
\hline $20-45$ anos & 331 & 77,2 & 53,2 & 1 \\
\hline \multicolumn{5}{|l|}{ Cor da pele } \\
\hline Não branca & 328 & 76,5 & 47,3 & $0,795^{\star}$ \\
\hline Branca & 101 & 23,5 & 59,4 & 1 \\
\hline \multicolumn{5}{|l|}{ Escolaridade materna } \\
\hline Até 10 anos completos & 267 & 62,2 & 46,4 & $0,827^{\star}$ \\
\hline 11 anos completos ou mais & 162 & 37,8 & 56,2 & 1 \\
\hline \multicolumn{5}{|l|}{ Renda materna } \\
\hline Sem renda & 164 & 38,2 & 53,7 & 1 \\
\hline 1 salário mínimo ou mais & 144 & 33,6 & 52,8 & 0,984 \\
\hline < 1 salário mínimo & 120 & 28,0 & 42,5 & $0,792^{*}$ \\
\hline \multicolumn{5}{|l|}{ Experiência prévia em AM } \\
\hline AM por $<6$ meses & 58 & 13,5 & 43,1 & $0,771^{\star}$ \\
\hline Primíparas & 219 & 51,0 & 47,9 & $0,857^{\star}$ \\
\hline AM por $\geq 6$ meses & 152 & 35,4 & 55,9 & 1 \\
\hline \multicolumn{5}{|l|}{ Vive com companheiro } \\
\hline Sim & 338 & 78,8 & 53,6 & 1 \\
\hline Não & 91 & 21,2 & 37,4 & $0,698^{*}$ \\
\hline \multicolumn{5}{|l|}{ Chefe da família } \\
\hline Companheiro & 282 & 65,9 & 54,6 & 1 \\
\hline Outra pessoa & 146 & 34,1 & 41,8 & $0,765^{\star}$ \\
\hline \multicolumn{5}{|l|}{ No de moradores } \\
\hline Até 4 moradores & 270 & 62,9 & 49,3 & 1 \\
\hline De 5 a 11 moradores & 159 & 37,1 & 51,6 & 1,047 \\
\hline \multicolumn{5}{|l|}{ Local de moradia } \\
\hline Bairro & 162 & 37,8 & 51,2 & 1 \\
\hline Comunidade & 267 & 62,2 & 49,4 & 0,965 \\
\hline
\end{tabular}

$\mathrm{RPb}=$ razão de prevalência bruta; $\mathrm{AME}=$ aleitamento materno exclusivo; $\mathrm{AM}=$ aleitamento materno; ${ }^{*} \mathrm{p}<0,20$.

no Rio de Janeiro, em $2008(40,7 \%)^{18}$. A prevalência de aleitamento materno exclusivo encontrada foi um pouco superior à verificada entre $2007 \mathrm{e}$ 2008 na mesma clientela da rede básica da cidade do Rio de Janeiro $(47,6 \%)^{19}$, podendo tanto representar um avanço decorrente das políticas desenvolvidas, ou refletir um maior envolvimento das unidades básicas que dispõem de Posto de Recebimento de Leite Humano Ordenhado na promoção do aleitamento materno exclusivo.

As mulheres com renda inferior a um salário mínimo apresentaram uma prevalência de amamentação exclusiva $23 \%$ inferior às mães que não tinham renda própria, que provavelmente eram sustentadas pelo companheiro ou pela família. Esse resultado se assemelha aos achados de Martins et al. ${ }^{20}$ e de Mascarenhas et al. ${ }^{21}$, que ao acompanharem coortes de nascimento observaram que a baixa renda familiar foi um fator associado à interrupção precoce do aleitamento materno exclusivo. Achado preocupante, na medida em que crianças de famílias de baixa renda são mais vulneráveis à morbimortalidade infantil, e a introdução precoce de outros alimentos pode potencializar este risco ${ }^{22}$.

Ter amamentado o último filho por período inferior a 6 meses se associou a uma menor prevalência de aleitamento materno exclusivo, de forma similar ao estudo de Pereira et al. ${ }^{23}$, realizado em 27 unidades básicas da cidade do Rio de Janeiro, onde a prevalência de amamentação exclusiva nos primeiros seis meses de vida foi $27 \%$ superior entre mulheres que haviam amamentado previamente por mais de seis meses. 
Tabela 2. Prevalência e razão de prevalência bruta de aleitamento materno exclusivo em menores de 6 meses segundo características da assistência pré-natal e ao parto, características do bebê e da assistência no hospital. Município do Rio de Janeiro, 2013.

\begin{tabular}{|c|c|c|c|c|}
\hline Características & $\mathbf{N}$ & $\%$ & AME (\%) & RPb AME \\
\hline \multicolumn{5}{|l|}{ No de consultas de pré-natal } \\
\hline 6 ou mais consultas & 379 & 88,3 & 52,5 & 1 \\
\hline 0 a 5 consultas & 46 & 10,7 & 32,6 & $0,609^{*}$ \\
\hline \multicolumn{5}{|c|}{ Recebeu orientação no PN sobre AM } \\
\hline Sim & 338 & 78,8 & 50,3 & 1,017 \\
\hline Não & 91 & 21,2 & 49,5 & 1 \\
\hline \multicolumn{5}{|l|}{ Forma de orientação no PN } \\
\hline \multicolumn{5}{|l|}{ Consulta } \\
\hline Sim & 253 & 59,0 & 49,8 & 0,954 \\
\hline Não & 176 & 41,0 & 50,6 & 1 \\
\hline \multicolumn{5}{|l|}{ Grupo de apoio } \\
\hline Sim & 168 & 39,2 & 53,6 & 1,130 \\
\hline Não & 261 & 60,8 & 47,9 & 1 \\
\hline \multicolumn{5}{|l|}{ Visita domiciliar } \\
\hline Sim & 19 & 4,4 & 47,4 & 0,936 \\
\hline Não & 410 & 95,6 & 50,2 & 1 \\
\hline \multicolumn{5}{|l|}{ Hospital de nascimento } \\
\hline Hospital Amigo da Criança & 118 & 27,6 & 55,1 & 1 \\
\hline Não credenciado & 309 & 72,4 & 48,2 & $0,875^{\star}$ \\
\hline \multicolumn{5}{|l|}{ Tipo de parto } \\
\hline Cesariana & 182 & 42,4 & 52,7 & 1,095 \\
\hline Normal & 247 & 57,6 & 48,2 & 1 \\
\hline \multicolumn{5}{|l|}{ Idade gestacional } \\
\hline Pré-termo & 41 & 9,6 & 41,5 & 0,813 \\
\hline A termo & 388 & 90,4 & 51,0 & 1 \\
\hline \multicolumn{5}{|l|}{ Peso ao nascer } \\
\hline$<2500 \mathrm{~g}$ & 26 & 6,1 & 46,2 & 0,916 \\
\hline $2500 \mathrm{~g}$ ou mais & 403 & 93,9 & 50,4 & 1 \\
\hline \multicolumn{5}{|l|}{ Primeira mamada } \\
\hline Após a primeira hora & 182 & 42,4 & 47,3 & 0,905 \\
\hline Na primeira hora & 247 & 57,6 & 52,2 & 1 \\
\hline \multicolumn{5}{|l|}{ Internação em unidade neonatal } \\
\hline Sim & 74 & 17,2 & 50,0 & 0,997 \\
\hline Não & 355 & 82,8 & 50,1 & 1 \\
\hline \multicolumn{5}{|l|}{ AME na alta hospitalar } \\
\hline Sim & 336 & 78,3 & 53,0 & 1 \\
\hline Não & 93 & 21,7 & 39,8 & $0,751^{*}$ \\
\hline
\end{tabular}

Experiências anteriores de amamentação bemsucedida podem não garantir, mas possivelmente interferem positivamente na decisão e na prática dos moldes preconizados de aleitamento materno com filhos posteriores ${ }^{24}$.

Não viver com o companheiro se associou a uma prevalência de aleitamento materno exclu- sivo $24 \%$ inferior. É importante que a mulher nutriz seja apoiada na prática da amamentação exclusiva por pessoas próximas, principalmente o companheiro, pois seu estímulo é o mais significativo para que a mulher possa amamentar ${ }^{25}$. De forma convergente ao presente estudo, Pereira et al. ${ }^{23}$ observaram que mulheres que viviam com 
Tabela 3. Prevalência e razão de prevalência bruta de aleitamento materno exclusivo em menores de 6 meses segundo o estilo de vida materno, as orientações sobre aleitamento materno recebidas na unidade básica e características do bebê. Município do Rio de Janeiro, 2013.

\begin{tabular}{|c|c|c|c|c|}
\hline Estilo de vida e orientações & $\mathbf{N}$ & $\%$ & $\operatorname{AME}(\%)$ & RPb AME \\
\hline \multicolumn{5}{|l|}{ Estuda } \\
\hline Sim & 44 & 10,3 & 52,3 & 1,045 \\
\hline Não & 384 & 89,7 & 50,0 & 1 \\
\hline \multicolumn{5}{|l|}{ Trabalho remunerado } \\
\hline $\operatorname{Sim}$ & 173 & 40,3 & 51,4 & 1,045 \\
\hline Não & 256 & 59,7 & 49,2 & 1 \\
\hline \multicolumn{5}{|l|}{ Fuma } \\
\hline $\operatorname{Sim}$ & 53 & 12,4 & 34,0 & $0,648^{*}$ \\
\hline Não & 376 & 87,6 & 52,4 & 1 \\
\hline \multicolumn{5}{|l|}{ Consome bebida alcoólica } \\
\hline Sim & 50 & 11,7 & 28,0 & $0,528^{*}$ \\
\hline Não & 379 & 88,3 & 53,0 & 1 \\
\hline \multicolumn{5}{|l|}{ Percepção da sua saúde } \\
\hline Ruim ou regular & 66 & 15,4 & 36,4 & $0,691^{*}$ \\
\hline Boa & 363 & 84,6 & 52,6 & 1 \\
\hline \multicolumn{5}{|l|}{ Orientação sobre AME } \\
\hline $\operatorname{Sim}$ & 355 & 82,9 & 52,7 & $1,424^{\star}$ \\
\hline Não & 73 & 17,1 & 37,0 & 1 \\
\hline \multicolumn{5}{|l|}{ Orientação sobre pega/posição } \\
\hline Sim & 272 & 63,4 & 53,3 & $1,196^{*}$ \\
\hline Não & 157 & 36,6 & 44,6 & 1 \\
\hline \multicolumn{5}{|l|}{ Orientação sobre ordenha } \\
\hline Não & 168 & 39,2 & 49,4 & 1 \\
\hline Sim, com as mãos & 242 & 56,4 & 52,5 & 1,062 \\
\hline Sim, com a bomba & 19 & 4,4 & 26,3 & $0,533^{*}$ \\
\hline \multicolumn{5}{|l|}{ Orientação sobre livre demanda } \\
\hline Sim & 238 & 76,5 & 52,1 & $1,197^{\star}$ \\
\hline Não & 101 & 23,5 & 43,6 & 1 \\
\hline \multicolumn{5}{|c|}{ Orientação sobre o não uso de mamadeira } \\
\hline Sim & 329 & 76,9 & 52,6 & $1,239^{*}$ \\
\hline Não & 99 & 23,1 & 42,4 & 1 \\
\hline \multicolumn{5}{|l|}{ Uso de chupeta } \\
\hline Sim & 192 & 55,1 & 40,1 & $0,686^{*}$ \\
\hline Não & 236 & 44,9 & 58,5 & 1 \\
\hline \multicolumn{5}{|l|}{ Sexo do bebê } \\
\hline Masculino & 232 & 54,1 & 49,6 & 0,977 \\
\hline Feminino & 197 & 45,9 & 50,8 & 1 \\
\hline \multicolumn{5}{|l|}{ Idade do bebê } \\
\hline$<2$ meses & 110 & 25,6 & 75,5 & 1 \\
\hline 2-3 meses e 29 dias & 176 & 41,0 & 48,9 & $0,648^{*}$ \\
\hline 4-5 meses e 29 dias & 143 & 33,3 & 32,2 & $0,426^{*}$ \\
\hline
\end{tabular}

$\mathrm{RPb}=$ razão de prevalência bruta; $\mathrm{AME}=$ aleitamento materno exclusivo; ${ }^{*} \mathrm{p}<0,20$.

o companheiro apresentaram aleitamento materno exclusivo $72 \%$ superior.

As orientações sobre aleitamento materno em grupos educativos e durante visitas domi- ciliares no pré-natal foram pouco frequentes e não se associaram ao aleitamento materno exclusivo, possivelmente porque estes espaços não vêm sendo aproveitados em seu potencial. Esses 
Tabela 4. Análise múltipla hierarquizada dos fatores associados ao aleitamento materno exclusivo entre mães de crianças menores de 6 meses assistidas em unidades básicas. Município do Rio de Janeiro, 2013.

\begin{tabular}{|c|c|c|c|}
\hline Características distais & RP ajustada de AME & IC95\% & p-valor \\
\hline \multicolumn{4}{|l|}{ Renda materna } \\
\hline Sem renda & 1 & & \\
\hline 1 salário mínimo ou mais & 1,033 & $0,856-1,248$ & 0,732 \\
\hline$<1$ salário mínimo & 0,771 & $0,609-0,975$ & 0,030 \\
\hline \multicolumn{4}{|l|}{ Experiência prévia de AM } \\
\hline AM por $<6$ meses & 0,728 & $0,540-0,981$ & 0,037 \\
\hline Primíparas & 0,840 & $0,699-1,010$ & 0,064 \\
\hline AM por $\geq 6$ meses & 1 & & \\
\hline \multicolumn{4}{|l|}{ Vive com companheiro } \\
\hline Sim & 1 & & \\
\hline Não & 0,761 & $0,585-0,991$ & 0,042 \\
\hline \multicolumn{4}{|l|}{ Características intermediárias } \\
\hline \multicolumn{4}{|l|}{ AME na alta hospitalar } \\
\hline Sim & 1 & & \\
\hline Não & 0,775 & $0,614-0,979$ & 0,032 \\
\hline \multicolumn{4}{|l|}{ Características proximais } \\
\hline \multicolumn{4}{|l|}{ Consumo de bebida alcoólica } \\
\hline Sim & 0,567 & $0,380-0,845$ & 0,005 \\
\hline Não & 1 & & \\
\hline \multicolumn{4}{|l|}{ Orientação sobre AME } \\
\hline Sim & 1,320 & $1,001-1,741$ & 0,049 \\
\hline Não & 1 & & \\
\hline \multicolumn{4}{|l|}{ Orientação sobre ordenha } \\
\hline Não & 1 & & \\
\hline Sim, com as mãos & 1,028 & $0,814-1,162$ & 0,760 \\
\hline Sim, com a bomba & 0,527 & $0,263-1,000$ & 0,050 \\
\hline \multicolumn{4}{|l|}{ Uso de chupeta } \\
\hline $\operatorname{Sim}$ & 0,739 & $0,616-0,887$ & 0,001 \\
\hline Não & 1 & & \\
\hline Idade do bebê em meses & 0,781 & $0,739-0,825$ & $<0,001$ \\
\hline
\end{tabular}

$\mathrm{RP}=$ razão de prevalência; $\mathrm{AME}$ = aleitamento materno exclusivo; $\mathrm{AM}=$ aleitamento materno.

resultados diferem dos encontrados no Rio de Janeiro $^{23}$, e em Pernambuco ${ }^{26}$, onde orientações em grupo e visitas domiciliares, respectivamente, se associaram a prevalências superiores de aleitamento materno exclusivo e mostraram-se fontes importantes de apoio à amamentação. Mães que recebem orientações em grupo se sentem mais assistidas pela diversidade de experiências compartilhadas e pela segurança proporcionada por este espaço, possibilitando-as a tomar decisões relativas ao aleitamento materno ${ }^{27}$.

A prevalência de amamentação exclusiva foi $22 \%$ menor entre as mães que não tiveram alta hospitalar em aleitamento materno exclusivo. Associação ainda de maior intensidade foi obser- vada em outro estudo realizado na cidade do Rio de Janeiro ${ }^{23}$, onde o aleitamento materno exclusivo na alta hospitalar dobrou a prevalência de amamentação exclusiva no primeiro semestre de vida. O oferecimento de complementos no hospital pode prejudicar o estabelecimento da lactação e a produção de leite adequada, dificultando $\mathrm{o}$ aleitamento materno exclusivo ${ }^{28}$.

A prevalência de aleitamento materno exclusivo entre as mães que consumiam bebida alcoólica foi $43 \%$ inferior. Chaves et al..$^{29}$ observaram em Minas Gerais que mulheres que relataram fazer uso de álcool e tabaco amamentaram seus filhos por menos tempo do que aquelas que não faziam uso destas substâncias. O Ministério da 
Saúde contraindica o uso de álcool durante o período de amamentação, pois a ingestão de doses iguais ou superiores a $0,3 \mathrm{~g} / \mathrm{kg}$ de peso pode reduzir a produção láctea, dificultando o aleitamento materno exclusivo. O consumo de álcool pela nutriz pode também modificar o aroma e o sabor do leite materno, levando à redução da sua ingestão pelo lactente ${ }^{30}$. O consumo elevado de álcool pela mãe pode inclusive gerar sonolência, atraso no crescimento e diminuição do ganho de peso no bebê $\hat{e}^{31}$.

A única orientação que se associou positivamente a este desfecho foi sobre a importância do aleitamento materno exclusivo por seis meses, que correspondeu a uma prevalência $32 \%$ superior de amamentação exclusiva, diferentemente do encontrado por Pereira et al. ${ }^{23}$. Este é um achado importante, pois o reflexo das orientações em aleitamento materno prestadas na atenção básica é tema pouco estudado, e o presente estudo foi o primeiro a encontrar uma associação positiva entre a orientação sobre o aleitamento materno exclusivo e a sua prática.

Um aspecto preocupante é que as orientações sobre pega e posição, relevantes para o manejo da amamentação, mas que requerem tempo e habilidade dos profissionais de saúde, foram menos abordadas do que informações sobre a importância do aleitamento materno exclusivo, sobre a amamentação em livre demanda e sobre o não uso de mamadeira. No entanto, a associação entre as orientações sobre pega e posição e o aleitamento materno exclusivo, observada por Pereira et al. ${ }^{23}$, não se confirmou no presente estudo.

A prevalência de aleitamento materno exclusivo entre as mães que receberam orientação sobre ordenha das mamas com uso de bomba foi quase a metade em relação às mães não orientadas. O uso de bombas de extração de leite pode acarretar problemas como traumas mamilares e monilíase, gerando dor, uma importante causa de desmame ${ }^{32}$. Esta variável precisa ser melhor investigada, pois foi identificado apenas um estudo que explorou a orientação sobre ordenha das mamas, sem distinguir o tipo, não tendo sido verificada associação com o aleitamento materno exclusivo $^{23}$.

O uso de chupeta se mostrou inversamente associado ao aleitamento materno exclusivo. Crianças que usavam chupeta foram amamentadas exclusivamente $26 \%$ menos do que as que não usavam. A repercussão do uso da chupeta sobre o aleitamento materno exclusivo tem sido mostrada por diversos estudos ${ }^{16}$. O uso de chupeta é prejudicial à amamentação pois pode acarretar confusão de bicos, reduzir a frequência das mamadas e diminuir a produção do leite materno. No entanto, a relação causal entre o uso de chupeta e o desfecho não é clara, pois não se sabe se o uso de chupeta é um marcador da interrupção do aleitamento materno exclusivo, ou se é uma causa da mesma. $\mathrm{O}$ uso de chupeta pode ainda estar relacionado à insegurança da mãe em amamentar, assim como a dificuldades e problemas vivenciados ao longo do processo da amamentação ${ }^{33}$.

A prevalência de aleitamento materno exclusivo diminuiu em $22 \%$ a cada mês de vida do bebê. À medida que aumenta a idade da criança a probabilidade da introdução de outros líquidos e alimentos também aumenta. Em estudo realizado em Barra Mansa, no Estado do Rio de Janeiro, o aleitamento materno exclusivo se reduziu em $1 \%$ a cada dia de vida da criança ${ }^{34}$ e na cidade do Rio de Janeiro a amamentação exclusiva foi reduzida em $17 \%$ a cada mês da criança ${ }^{23}$.

Limitações do presente estudo devem ser apontadas. As mães podem não ter se recordado de informações prestadas há mais tempo, como as relativas ao período da gravidez, portanto a falta de associação do desfecho com a assistência pré-natal poderia também ser atribuída a viés de memória. Outra questão a ser ressalvada é a população do estudo. Unidades de saúde com Posto de Recebimento de Leite Humano Ordenhado provavelmente estão mais engajadas na promoção do aleitamento materno, portanto as orientações prestadas nestas unidades podem não refletir a prática do conjunto das unidades primárias da cidade do Rio de Janeiro.

Conclui-se que a orientação sobre a importância do aleitamento materno exclusivo por seis meses se associou a uma maior prevalência desta prática. Entretanto, orientações sobre o manejo da amamentação não mostraram influência sobre o aleitamento materno exclusivo no contexto estudado, sinalizando que a frequência e a qualidade destas orientações precisam ser aprimoradas. As crianças de mães de baixa renda, sem companheiro e que consumiam bebidas alcoólicas, conhecidos fatores de risco para desfechos infantis ${ }^{31,35}$, apresentaram uma menor prevalência de aleitamento materno exclusivo, contribuindo assim para a perpetuação das desigualdades em saúde nos grupos de maior vulnerabilidade.

Recomenda-se que a rede primária de saúde preste orientações às gestantes e mães sobre os benefícios e o manejo do aleitamento materno. Os profissionais de saúde devem possuir habilidades de aconselhamento às mães ${ }^{36} \mathrm{e}$ capacitação 
em estratégias de promoção e apoio ao aleitamento materno na atenção primária ${ }^{9}$, para que suas orientações sejam efetivas e as mães possam se sentir seguras e superar possíveis dificuldades que surjam no processo da amamentação, contribuindo, assim, para a prática do aleitamento materno exclusivo, cuja mediana em nosso país ainda é baixa. Recomenda-se também que mais estudos sejam conduzidos sobre o tema, para que se conheça melhor as orientações em aleitamento materno e a forma como vêm sendo prestadas na atenção primária.

\section{Colaboradores}

JS Alves participou da coleta de dados, foi a responsável principal pela análise e interpretação dos dados, e pela redação do artigo. MIC Oliveira participou da concepção do estudo, da análise e interpretação dos dados, da redação do artigo e da aprovação da versão final do artigo. RVVF Rito participou da concepção do estudo, da redação do artigo e da aprovação da versão final do artigo. 


\section{Referências}

1. Horta BL, Mola CL, Victora CG. Long-term consequences of breastfeeding on cholesterol, obesity, systolic blood pressure and type 2 diabetes: a systematic review and meta-analysis. Acta Paediatr 2015; 104(467):30-37.

2. Boccolini CS, de Carvalho ML, Oliveira MI, PérezEscamilla R. Breastfeeding during the first hour of life and neonatal mortality. J Pediatr (Rio J) 2013; 89(2):131-136.

3. Chowdhury R, Sinha B, Sankar MJ, Taneja S, Bhandari1 $\mathrm{N}$, Rollins N, Bahl R, Martines J. Breastfeeding and maternal health outcomes: a systematic review and meta-analysis. Acta Paediatr 2015; 104(467):96-113.

4. World Health Organization (WHO). Dept. of Child and Adolescent Health and Development. Indicators for assessing infant and young child feeding practices: conclusions of a consensus meeting held 6-8 November 2007 in Washington D.C., USA. Geneva: WHO; 2008.

5. Brasil. Ministério da Saúde (MS). Centro Brasileiro de Análise e Planejamento. Pesquisa Nacional de Demografia e Saúde da Criança e da Mulher - PNDS 2006: dimensões do processo reprodutivo e da saúde da criança. Brasília: Editora do Ministério da Saúde; 2009.

6. Venancio SI, Escuder MML, Saldiva SRDM, Giugliani ERJ. A prática do aleitamento materno nas capitais brasileiras e Distrito Federal: situação atual e avanços. J Pediatr (Rio J) 2010; 86(4):317-324.

7. Rea MF. Reflexões sobre a amamentação no Brasil: de como passamos a 10 meses de duração. Cad Saude Publica 2003: 19(1):37-45.

8. World Health Organization (WHO), United Nations Children's Fund (UNICEF). Baby-Friendly Hospital Initiative. Revised, updated and expanded for integrated care. Geneva: WHO; 2009.

9. Oliveira MIC, Camacho LAB, Souza IEO. Promoção, proteção e apoio à amamentação na atenção primária à saúde no Estado do Rio de Janeiro, Brasil: uma política de saúde pública baseada em evidência. Cad Saude Publica 2005; 21(6):1901-1910.

10. Chung M, Raman G, Trikalinos T, Lau J, Ip S. Interventions in primary care to promote breastfeeding: an evidence review for the U.S. Preventive Services Task Force. Ann Intern Med 2008; 149(8):565-582.

11. Renfrew MJ, McCormick FM, Wade A, Quinn B, Dowswell T. Support for healthy breastfeeding mothers with healthy term babies. Cochrane Database Syst Rev 2012; (5):CD001141.

12. von Seehausen MP, Oliveira MIC, Boccolini, CS. Fatores associados ao aleitamento cruzado. Cien Saude Colet 2015; 22(5):1673-1682.

13. Pellegrine JB, Koopmans FF, Pessanha HL, Rufino CG, Farias HPS. Educação popular em saúde: doação de leite humano em comunidade do Rio de Janeiro, Brasil. Interface 2014; 18 (Supl. 2):1499-1506.

14. Cochran WG. Sampling Techniques. In: Wiley Series. Probability and Statistics. Indianapolis: Ed. IE-WILEY; 1977.

15. Victora CG, Huttly SR, Fuchs SC, Olinto MT. The role of conceptual frameworks in epidemiological analysis: a hierarchical approach. Int J Epidemiol 1997; 26(1):224-227.
16. Boccolini CS, Carvalho ML, Oliveira MI. Factors associated with exclusive breastfeeding in the first six months of life in Brazil: a systematic review. Rev Saude Publica [serial on the Internet] 2015 [cited 2016 Apr 18]; 49:91[15 p.]. Available from: http://www.ncbi.nlm. nih.gov/pmc/articles/PMC4687824/

17. Barros AJD, Hirakata VN. Alternatives for logistic regression in cross-sectional studies: an empirical comparison of models that directly estimate the prevalence ratio. BMC Med Res Methodol [serial on the Internet] 2003 [cited 2016 Apr 18]; 3:21[13 p.]. Available from: http://www.biomedcentral.com/147-2288/3/21.

18. Brasil. Ministério da Saúde (MS). II Pesquisa de Prevalência de Aleitamento Materno nas Capitais Brasileiras e Distrito Federal. Brasília: Editora do Ministério da Saúde; 2009.

19. Rito RVVF, Oliveira MIC, Brito AS. Grau de cumprimento dos Dez Passos da Iniciativa Unidade Básica Amiga da Amamentação e sua associação com a prevalência de aleitamento materno exclusivo. J Pediatr (Rio J) 2013; 89(5):477-484.

20. Martins CC, Vieira GO, Vieira TO, Mendes CM. Fatores de riscos maternos e de assistência ao parto para interrupção precoce do aleitamento materno exclusivo: estudo de coorte. Rev Baiana Saude Publica 2011; 35(Supl. 1):167-168.

21. Mascarenhas MLW, Albernaz EP, Silva MB, Silveira RB. Prevalência de aleitamento materno exclusivo nos 3 primeiros meses de vida e seus determinantes no Sul do Brasil. J Pediatr (Rio J) 2006; 82(4):289-294.

22. Horta BL, Gigante DP, Candiota JS, Barros FC, Victora CG. Monitoring mortality in Pelotas birth cohort from 1982 to 2006, Southern Brazil. Rev Saude Publica 2008; 42(2):108-114.

23. Pereira RSV, Oliveira MIC, Andrade CLT, Brito AS, Fatores associados ao aleitamento materno exclusivo: o papel do cuidado na atenção básica. Cad Saude Publica 2010; 26(12):2343-2354.

24. Faleiro FTV, Trezza EMC, Carandina L. Factors influencing breastfeeding decision and duration. Rev Nutr 2006; 19(5):623-630.

25. Giugliani ERJ. Breast-feeding: how and why to promote it. J Pediatr (Rio J) 1994; 70(3):138-151.

26. Coutinho SB, Lira PIC, Lima MC, Ashworth A. Comparison of the effect of two systems for the promotion of exclusive breastfeeding. Lancet 2005; 366(9491):1094-1100.

27. Hoddinott P, Phil M, Chalmers M, Pill R. One-toone or group-based peer support for breastfeeding? Women's perceptions of a breastfeeding peer coaching intervention. Birth 2006; 33(2):139-146.

28. Carvalhaes MABL, Corrêa CRH. Identificação de dificuldades no início do aleitamento materno mediante aplicação de protocolo. J Pediatr (Rio J) 2003; 79(1):1320.

29. Chaves RG, Lamounier JA, César CC. Factors associated with duration of breastfeeding. J Pediatr (Rio J) 2007; 83(3):241-246.

30. Brasil. Ministério da Saúde (MS). Amamentação e uso de medicamentos e outras substâncias. Brasília: Editora do Ministério da Saúde; 2010. 
31. Kachani AT, Okuda LS, Barbosa ALR, Brasiliano S, Hochgraf PB. Aleitamento Materno: quanto o álcool pode influenciar na saúde do bebê? Pediatria (São Paulo) 2008; 30(4):249-256.

32. Giugliani ERJ. Problemas comuns na lactação e seu manejo. J Pediatr (Rio J) 2004; 80(5):147-154.

33. Jaafar SH, Jahanfar S, Angolkar M, Ho JJ. Effect of restricted pacifier use in breastfeeding term infants for increasing duration of breastfeeding. Cochrane Database Syst Rev 2012; 7:CD007202.

34. Alves ALN, Oliveira MIC, Moraes JR. BreastfeedingFriendly Primary Care Unit Initiative and the relationship with exclusive breastfeeding. Rev Saude Publica 2013; 47(6):1130-1140.

35. França E, Souza JM, Guimarães MDC, Goulart EMA, Colosimo E, Antunes CMF. Associação entre fatores sócio-econômicos e mortalidade infantil por diarréia, pneumonia e desnutrição em região metropolitana do Sudeste do Brasil: um estudo caso-controle. Cad Saude Publica 2001; 17(6):1437-1447.

36. Bueno LGS, Teruya KM. Aconselhamento em amamentação e sua prática. J Pediatr (Rio J) 2004; 80(5 Supl.):126-130.

Artigo apresentado em 19/04/2016

Aprovado em 23/06/2016

Versão final apresentada em 25/06/2016 